The University of Southern Mississippi

The Aquila Digital Community

Faculty Publications

$11-1-2005$

\title{
Particular Solutions for Axisymmetric Helmholtz-Type Operators
}

A.S. Muleshkov

University of Nevada

M.A. Golberg

C.S. Chen

University of Southern Mississippi, CS.Chen@usm.edu

Follow this and additional works at: https://aquila.usm.edu/fac_pubs

Part of the Mathematics Commons

\section{Recommended Citation}

Muleshkov, A., Golberg, M., Chen, C. (2005). Particular Solutions for Axisymmetric Helmholtz-Type Operators. Engineering Analysis With Boundary Elements, 29(11), 1066-1076.

Available at: https://aquila.usm.edu/fac_pubs/2615

This Article is brought to you for free and open access by The Aquila Digital Community. It has been accepted for inclusion in Faculty Publications by an authorized administrator of The Aquila Digital Community. For more information, please contact Joshua.Cromwell@usm.edu. 


\title{
Particular Solutions for Axisymmetric Helmholtz-Type Operators
}

\author{
A.S. Muleshkov ${ }^{*}$ M.A. Golberg $†$ C.S. Chen ${ }^{\ddagger}$
}

\begin{abstract}
In this paper, we consider the solution of the axisymmetric heat equation with axisymmetric data in an axisymmetric domain in $R^{3}$. To solve this problem, we remove the time-dependence by various transform or time-stepping methods. This converts the problem to one of a sequence of modified inhomogeneous Helmholtz equations. Generalizing previous work, we consider solving these equations by boundary-type methods. In order to do this, one needs to subtract off a particular solution, so that one obtains a sequence of modified homogeneous Helmholtz equations. We do this by modifying the usual Dual Reciprocity Method (DRM) and approximating the right-hand sides by Fourier-polynomials or bivariate polynomials. This inevitably leads to analytical solving a sequence of ordinary differential equations (ODEs.) The analytic formulas and their precision are checked using MATHEMATICA. In fact, by using an infinite precision technique, the particular solutions can be obtained with infinite precision themselves. This work will form the basis for numerical algorithms for solving axisymmetric heat equation.
\end{abstract}

\section{Introduction}

Over the past two decades, there has been increasing interest in using boundary-type methods for solving various boundary-value problems in physics and engineering [?]. Such methods typically involve the Boundary Integral Method (BIM), the Method of Fundamental Solutions (MFS), and other Trefftz methods. Most of these methods have been restricted to elliptic partial differential equations (PDEs) that arise in potential theory [?], steady state heat transfer [?], electromagnetic and acoustic scattering [?], and elastostatics [?]. However, increasingly, scientists are becoming interested in solving time-dependent problems, such as those that occur in transient heat flow [?], wave scattering [?], reaction-diffusion problems [?], and the Navier-Stokes equations [?]. These

\footnotetext{
*Department of Mathematical Sciences, University of Nevada, Las Vegas, NV 89154-4020, USA

$\dagger 7385$ Edna Ave., Las Vegas, NV 89117, USA

${ }^{\ddagger}$ Department of Mathematics, University of Southern Mississippi, Hattiesburg, MS 39406, USA
} 
problems require solving parabolic or hyperbolic PDEs. Although, it is possible to reformulate these PDEs as integral equations using time-dependent fundamental solutions, there are a number of difficulties associated with this approach. If there are non-zero initial conditions on non-zero source terms, then direct boundary integral reformulations produce domain integrals that can be very difficult to evaluate with high accuracy [?]. To reformulate non-linear problems, one usually needs to use some type of iterative scheme which again leads to evaluating domain integrals [?]. For hyperbolic equations, particularly in $3 \mathrm{D}$, the fundamental solutions are distributions rather than functions, so it is difficult to give a clear mathematical interpretation to these integral equations. To overcome these difficulties, it is often more appropriate to reformulate the time-dependent problem as a sequence of time-independent elliptic problems. This can be done in a number of ways using Laplace or Laguerre transformations [?], convolution quadrature or various time-stepping schemes [?, ?]. However, this again leads to evaluation of a large number of domain integrals. These typically arise from source terms that occur as a consequence of converting the time-dependent problem into a sequence of time-independent ones. Over the past twenty years, a number of strategies have been developed to alleviate these difficulties. Perhaps, the most popular method in the engineering literature has been the Dual Reciprocity Method (DRM) [?, ?]. In this approach, source terms are approximated by finite sums of basis functions. A particular solution to the governing differential equation is then determined for each basis function. These are then summed to obtain an approximate particular solution to the given differential equation. This approximate particular solution then can be subtracted off leading to a homogeneous differential equation that can then be solved by a boundary integral or Trefftz method.

There are two mathematical difficulties associated with this technique. The first is the necessity of finding accurate approximation to the source term using the chosen basis functions, particularly for 3D problems. The second is the requirement that the particular solution be found analytically, rather than numerically. Hence, it is necessary to choose basis functions carefully in order to circumvent these difficulties. For radially symmetric operators, radial basis functions (rbfs) have been the basis functions of choice in the engineering literature. However, for 3D problems, rbf approximation can be costly and somewhat unstable. These difficulties are less pronounced for $2 \mathrm{D}$ problems, so, it is of some interest to see if properties of the boundary value problems can be used to mitigate the dimensional difficulties. A commonly occurring property is that of axisymmetry when both the domain and data are axisymmetric. In this case, when using boundary integral methods, a further dimensionality reduction occurs, since, in 3D, the 2D boundary integral equation can be reduced to a 1D integral equation over the generator of the solution domain. Unfortunately, no such simple dimensionality reduction occurs when computing particular solutions using radial basis functions. In this case, one converts the governing PDE to cylindrical co-ordinates in order to account for the axisymmetry, then the transformed PDE no longer is radially symmetric, so, analytic expressions for the particular solutions cannot be easily found. One method for dealing with this issue is to use the original 3D formulation, find a particular solution using 3D approximation, 
and then obtain an axisymmetric particular solution by integrating with respect to the azimuthal angle. This approach has been used by Šarler [?] and Wang [?] for Laplace and Helmholtz-type equations. However, this seems to counteract the dimensionality reduction provided by the axisymmetry, since it still requires 3D approximation and often substantial numerical integration. As we showed in [?, ?] for the Poisson equation, these problems can be overcome to some extent by using polynomial rather than rbf approximation. In this case, only $2 \mathrm{D}$ approximation is required, and it was shown in [?] that analytical particular solutions could be obtained for the axisymmetric Poisson equation. When compared to the 3D rbf approach, a substantial reduction in computing time was found.

It is the purpose of this paper to extend the above technique to find analytical particular solutions for Helmholtz-type operators. This work is primarily devoted to developing boundary type algorithms for solving time-dependent PDEs.

This work has been primarily motivated by Wang's Ph.D. thesis [?] as was the motivation for our work for the Poisson equation [?]. Other authors [?, ?] have looked at such problems, however, their calculations of particular solutions used 3D approximation and do not appear to be mathematically correct.

The issue in references [?, ?, ?] centers around the possible incorrect use of Kansa's method for calculating approximate particular solutions to the differential equation $L u=$ $f$. In this method, one chooses $N$ arbitrary functions $\psi_{j}, 1 \leq j \leq N$, and then defines basis functions

$$
\phi_{j}=L \psi_{j}, \quad 1 \leq j \leq N .
$$

Using the functions $\phi_{j}$, one can obtain an approximation $\hat{f}$ to $f$ in the form

$$
\hat{f}=\sum_{j=1}^{N} a_{j} \phi_{j}
$$

where $a_{j}$ are obtained by collocating the expression for $\hat{f}$ at $N$ arbitrary points in the solution domain. Then, one can get an approximate particular solution

$$
\hat{u}=\sum_{j=1}^{N} a_{j} \psi_{j}
$$

It is easily checked, using the definition of $\phi_{j}$ and the linearity of $L$, that $L \hat{u}=\hat{f}$. This was the method used in $[?, ?, ?]$. However, unless the functions $\psi_{j}$ satisfy very special properties, there is no guarantee that the coefficients $a_{j}$ are well defined, i.e. the collocation problem has unique solution. These properties were not established in $[?, ?, ?]$.

The paper is organized as follows. In Section 2, we consider initial boundary value problems for a class of diffusion equations on a bounded axisymmetric domain on $\mathbb{R}^{3}$ with axisymmetric initial and boundary data. By removing the time dependence, we convert 
the initial boundary value problem to a sequence of axisymmetric modified Helmholtz equations. We then consider the Method of Particular Solutions (MPS) as a method for solving such equations. To maintain the efficiency for boundary-type methods, it is necessary to be able to compute axisymmetric particular solutions. In Section 3 we briefly review some previous methods for doing this based on 3D interpolation.

As shown in [?], it is probably more efficient to use 2D approximation of the source term. For this, we consider two types of approximation, a Fourier polynomial-type approximation in Section 4 and a 2D polynomial approximation in Section 5. For these approximations, we are able to obtain analytic particular solutions based on a fundamental Lemma for calculating particular solutions to an inhomogeneous Bessel equation. The final result depends on an additional combinatorial Lemma that is proven in an appendix.

We conclude the paper with some directions for future research.

\section{The Axisymmetric Diffusion Equation}

To motivate the results in Sections 4 and 5, we consider the initial boundary value problem (IBVP)

$$
\begin{aligned}
\Delta u(P, t)-c u(P, t) & =\frac{\partial u}{\partial t}(P, t), \quad P \in D, \\
u(P, 0) & =f(P), \quad P \in D,
\end{aligned}
$$

with boundary condition

$$
B u(P, t)=g(P, t), \quad P \in S
$$

where $\Delta$ denotes the Laplacian and $D$ is a bounded domain in $\mathbb{R}^{3}$ with boundary $S$, assumed to be piece-wise smooth. The function $g(P, t)$ is piece-wise smooth boundary function. The operator $B$ has the form

$$
B u(P, t)=\alpha(P, t)+\beta(P, t) u(P, t)+\gamma(P, t) \frac{\partial u}{\partial t}(P, t)
$$

where $\alpha(P, t), \beta(P, t)$, and $\gamma(P, t)$ are prescribed piece-wise continuous functions. $\partial u / \partial n$ is the outward normal derivative of $u$ at point $P \in S$. This includes various boundary conditions on various portions of the boundary $S$ : Dirichlet with $\beta(P, t)=1$ and $\gamma(P, t)=$ 0 , Neumann with $\beta(P, t)=0$ and $\gamma(P, t)=1$, and Robin in general. Suppose that $D$ is an axisymmetric domain that is formed as a solid of revolution by rotating a plane region $\Omega$ with boundary $L$ about the z-axis as shown on Figure ??. Also assume that the data

Figure 1: Generator of simple connect domain. 
$(f, g, \alpha, \beta$, and $\gamma)$ is axisymmetric, as well. Then, introducing cylindrical co-ordinates $(r, \theta, z)$, the boundary value problem (??) - (??) becomes

$$
\begin{gathered}
\frac{1}{r^{2}} \frac{\partial^{2} u(P, t)}{\partial r^{2}}+\frac{1}{r} \frac{\partial u(P, t)}{\partial r}+\frac{\partial^{2} u(P, t)}{\partial z^{2}}-c u(P, t)=\frac{\partial u(P, t)}{\partial t}, \quad P \in D, \\
u(P, 0)=f(P), \quad P \in D
\end{gathered}
$$

and

$$
B u(P, t)=g(P, t), \quad P \in S,
$$

where $f, g, \alpha, \beta$, and $\gamma$ in (??) are functions only of $r$ and $z$. For convenience, we denote the axisymmetric Laplacian on the left hand side of (??) as

$$
\frac{1}{r^{2}} \frac{\partial^{2} u(P, t)}{\partial r^{2}}+\frac{1}{r} \frac{\partial u(P, t)}{\partial r}+\frac{\partial^{2} u(P, t)}{\partial z^{2}} \equiv \Delta_{r, z} u(P, t)
$$

To solve the boundary problem (??) - (??) by boundary-type methods, we first remove the time-dependence in order to reduce the boundary value problem to a sequence of timeindependent problems. This can be done in a number of ways. Here, we consider two possibilities, the Laplace Transform and finite differencing in time. Other approaches are possible and can be found in [?].

For the Laplace transform, we let $f(t)$ be a piece-wise continuous function of exponential growth on $[0,+\infty)$. Then, the Laplace transform $\hat{f}$ of $f$ is defined as

$$
\hat{f}(s)=\int_{0}^{\infty} e^{-s t} f(t) d t
$$

which is defined for all $s$ sufficiently large.

To solve the IBVP (??) - (??) for the diffusion equation, we take the Laplace transform of $u$ giving $\hat{u}$ as the solution of

$$
\Delta_{r, z} \hat{u}(P, s)-s \hat{u}(P, s)-c \hat{u}(P, s)=-f(P)
$$

where

$$
u(P, 0)=f(P) .
$$

Defining $\lambda^{2}=s+c, \hat{u}$ satisfies

$$
\Delta_{r, z} \hat{u}(P, s)-\lambda^{2} \hat{u}(P, s)=-f(P)
$$

Similarly, taking the Laplace transform of the boundary condition (??) gives

$$
B \hat{u}(P, s)=\hat{g}(P, s)
$$

where

$$
\hat{g}(P, s)=\int_{0}^{\infty} e^{-s t} g(P, t) d t .
$$


Thus, $\hat{u}(P, s)$ satisfies the BVP for the inhomogeneous modified Helmholtz equation (??) with boundary conditions (??). For numerical purposes, one solves (??) - (??) for a sequence of values $s,\left\{s_{n}\right\}_{n=1}^{m}$, and then applies a numerical inversion formula to the sequence $\left\{s_{n}\right\}$. Unfortunately, this could be problematic, as the numerical inversion of the Laplace transform is an ill-posed problem. Despite this, many inversion algorithms have appeared in the literature and an algorithm by Stehfest [?] has found some success in the solution of diffusion problems.

\subsubsection{Time-differencing}

As an alternative of Laplace Transform, a variety of time-differencing methods has been proposed to solve the IBVP (??) - (??). Among these are $\theta$ methods, time-splitting, and methods based on A-stable multi-step methods for ordinary differential equations (ODEs). As $\theta$ methods appear to be the most popular, we shall restrict our discussion on them. For this, define the mesh $t_{n}=n \tau, n \geq 0$. For $t_{n} \leq t \leq t_{n+1}, u(P, t)$ is approximated by

$$
u(P, t) \simeq \theta u\left(P, t_{n+1}\right)+(1-\theta) u\left(P, t_{n}\right)
$$

and

$$
\Delta_{r, z} u(P, t)=\theta \Delta_{r, z} u\left(P, t_{n+1}\right)+(1-\theta) \Delta_{r, z} u\left(P, t_{n}\right)
$$

where $0<\theta \leq 1$ and

$$
u_{t}(P, t)=\frac{u\left(P, t_{n+1}\right)-u\left(P, t_{n}\right)}{\tau}
$$

Using (??) and denoting the resulting approximation to $u\left(P, t_{n}\right)$ by $u_{n}$, then $u_{n}$ satisfies

$$
\theta \Delta_{r, z} u_{n+1}+(1-\theta) \Delta_{r, z} u_{n}-c\left(\theta u_{n+1}+(1-\theta) u_{n}\right)=\frac{u_{n+1}-u_{n}}{\tau}
$$

Rearranging (??) gives

$$
\Delta_{r, z} u_{n+1}-\frac{u_{n+1}}{\theta \tau}-c u_{n+1}=\frac{c(1-\theta) u_{n}}{\theta}-\frac{u_{n}}{\theta \tau}-\frac{(1-\theta) \Delta_{r, z} u_{n}}{\theta}
$$

For $\theta=1$, we get the backward Euler method with

$$
\Delta_{r, z} u_{n+1}-\frac{u_{n+1}}{\tau}-c u_{n+1}=-\frac{u_{n}}{\tau}
$$

Defining $\lambda^{2}=c+1 / \tau,(? ?)$ is of the form

$$
\Delta_{r, z} u_{n+1}-\lambda^{2} u_{n+1}=-\frac{u_{n}}{\tau}
$$

with initial condition

$$
u(P, 0)=f(P)
$$

and boundary condition

$$
B u_{n+1}=g_{n+1}=g\left(P, t_{n+1}\right)
$$


so again, numerically, the IBVP (??) - (??) is reduced to solving a sequence of BVPs for the inhomogeneous modified Helmholtz equation.

For $\theta=1 / 2$, we get the Crank-Nicholson scheme

$$
\begin{gathered}
\Delta_{r, z} u_{n+1}-\frac{2 u_{n+1}}{\tau}-c u_{n+1}=c u_{n}-\Delta_{r, z} u_{n}-\frac{2 u_{n}}{\tau} \\
u_{0}(P)=f(P) \\
B u_{n+1}=g_{n+1}=g\left(P, t_{n+1}\right)
\end{gathered}
$$

Again, letting $\lambda^{2}=c+2 / \tau, u_{n}$ satisfy a sequence of modified Helmholtz equations.

\section{Method of Particular Solutions}

In order to solve the inhomogeneous modified Helmholtz equation by boundary-type methods, it is necessary to eliminate the inhomogenuity in (??) and (??). This can be done by subtracting off a particular solution to equations (??) and (??). In fact, writing equations (??) and (??) in the form

$$
\Delta_{r, z} u-\lambda^{2} u=h
$$

we obtain a particular solution $\hat{u}_{p}$ from

$$
\Delta_{r, z} \hat{u}_{p}-\lambda^{2} \hat{u}_{p}=h
$$

where $\hat{u}_{p}$ does not have to satisfy the boundary conditions. Hence, defining

$$
v=u-\hat{u}_{p}
$$

it is easily shown that $v$ satisfies

$$
\Delta_{r, z} v-\lambda^{2} v=0
$$

and the boundary condition

$$
B v=g-B \hat{u}_{p}
$$

Assuming that the boundary data, $g$, and initial condition, $f$, are axisymmetric, all the data in (??) and the boundary condition are axisymmetric, as well. Hence, to preserve the axisymmetry in the BVP (??) - (??), we need a particular solution which is axisymmetric, as well.

In the engineering literature, the most popular way for obtaining particular solutions is to approximate the right-hand side, $h$, by a finite sequence $\left\{\varphi_{k}\right\}_{k=1}^{n}$, that is

$$
h \simeq \sum_{k=1}^{n} a_{k} \varphi_{k}
$$


Then an approximate particular solution $\hat{u}_{p}$ is given by

$$
\hat{u}_{p}=\sum_{k=1}^{n} a_{k} \Psi_{k}
$$

where $\Psi_{k}$ satisfies

$$
\Delta_{r, z} \Psi_{k}-\lambda^{2} \Psi_{k}=\varphi_{k} .
$$

As we will show, this can be rather difficult, because radial basis functions, that have been traditionally used, appear not to be feasible for axisymmetric Helmholtz equation. As a consequence, previous authors [?, ?, ?] have taken the alternative approach of using the original 3D Laplacian, finding particular solutions to it, and then integrating over the azimuthal angle. Unfortunately, closed form solutions cannot be obtained in this manner. Also, this approach requires 3D approximation to the right-hand side, $h$, which appear to defeat the dimensionality reduction provided by the axisymmetry. In our previous paper [?], we showed how to overcome this difficulty by using polynomials rather than radial basis functions. In that case, it was shown that considerable efficiency was obtained by using polynomial approximation of $h$. In this paper, we show how to extend the approach given in [?] to the axisymmetric modified Helmholtz equation. In order to be able to construct particular solutions to (??), we will use two forms of approximation to the right-hand side, $h$, a Fourier polynomial of $r$ and $z$ and a bivariate polynomial approximation to $h$.

\section{Fourier Polynomial Approximation}

The first type of approximation we use for $h(r, z)$ in (??) is a Fourier-polynomial approximation of the form

$$
h(r, z)=\sum_{m=0}^{\infty} h_{m}(r) e^{i m z}
$$

For practical purposes, $h(r, z)$ in (??) is truncated as

$$
h(r, z) \simeq \hat{h}(r, z)=\sum_{m=0}^{M} h_{m}(r) e^{i m z}
$$

where $M$ is chosen sufficiently large. Actual details of the method will be given in future work.

The approximate particular solution $\hat{u}_{p}$ is given by

$$
\hat{u}_{p}(r, z)=\sum_{m=0}^{M} \Psi_{m}(r) e^{i m z} .
$$


Then, substitution of (??) into the modified Helmholtz equation gives $\left\{\Psi_{m}\right\}_{m=0}^{M}$ as the solutions to

$$
\frac{d^{2}}{d r^{2}} \Psi_{m}+\frac{1}{r} \frac{d \Psi_{m}}{d r}-m^{2} \Psi_{m}-\lambda^{2} \Psi_{m}=h_{m}(r)
$$

where $m=0,1,2, \ldots, M$. Next, expanding $h_{m}(r)$ as

$$
h_{m}(r)=\sum_{k=0}^{\infty} g_{k, m}(r) r^{k}
$$

we approximate $h_{m}(r)$ by

$$
h_{m}(r) \simeq \hat{h}_{m}(r)=\sum_{k=0}^{K} g_{k, m} r^{k}
$$

where again $K$ is chosen sufficiently large. We then look for an approximate solution in the form

$$
\Psi_{m}(r)=\sum_{k=0}^{K} \Psi_{k, m} h_{k, m}
$$

Substituting $\Psi_{m}(r)$ into (??) and taking (??) into consideration, one obtains the following equations for $\left\{\Psi_{k, m}\right\}$

$$
\frac{d^{2}}{d r^{2}} \Psi_{k, m}+\frac{1}{r} \frac{d \Psi_{k, m}}{d r}-\lambda_{m} \Psi_{k, m}=r^{k}
$$

where $0 \leq k \leq K, 0 \leq m \leq M$, and $\lambda_{m}=m^{2}+\lambda^{2}$.

In order to obtain $\left\{\Psi_{k, m}\right\}$, it suffices to prove the following lemma.

Lemma 1 A particular solution to the ordinary differential equation

$$
y^{\prime \prime}+\frac{1}{x} y^{\prime}-\mu y=c x^{m}
$$

where $\mu \neq 0, c$ is a constant, and $m$ is a non-negative integer, is given by

$$
y(x)=c \sum_{s=1}^{\infty} \frac{(m ! !)^{2}}{(m+2 s) ! !^{2}} x^{m+2 s} \mu^{s-1}
$$

(Here $m$ !! means the product of all positive integers that are less than or equal to $m$ and have the same remainder as $m$ when divided by 2, e.g. $5 ! !=1 \times 3 \times 5=15,8$ !! = $2 \times 4 \times 6 \times 8=384$. More generally $(2 n) ! !=2^{n} n !$ and $(2 n-1) ! !=(2 n) ! /\left(2^{n} n !\right)$. Also, by separate definition, $2 ! !=2,0 ! !=1,1 ! !=1$, and $(-1) ! !=1$.) 
Proof. Calculating $y_{p}^{\prime}$ and $y_{p}^{\prime \prime}$ from (??) and then replacing the dummy integer variable $s$ by $s+1$, we get

$$
\begin{aligned}
y_{p}^{\prime} & =c \sum_{s=1}^{\infty} \frac{m ! !^{2}(m+2 s)}{(m+2 s) !^{2}} x^{m+2 s-1} \mu^{s-1} \\
y_{p}^{\prime \prime} & =c \sum_{s=1}^{\infty} \frac{m !^{2}(m+2 s)(m+2 s-1)}{(m+2 s) ! !^{2}} x^{m+2 s-2} \mu^{s-1} \\
& =c \sum_{s=1}^{\infty} \frac{m ! !^{2}(m+2 s)^{2}}{(m+2 s) !^{2}} x^{m+2 s-2} \mu^{s-1}-c \sum_{s=1}^{\infty} \frac{m ! !^{2}(m+2 s)}{(m+2 s) ! !^{2}} x^{m+2 s-2} \mu^{s-1} \\
& =c \sum_{s=1}^{\infty} \frac{m ! !^{2}}{(m+2 s-2) ! !^{2}} x^{m+2 s-2} \mu^{s-1}-\frac{c}{x} \sum_{s=1}^{\infty} \frac{m ! !^{2}(m+2 s)}{(m+2 s) ! !^{2}} x^{m+2 s-1} \mu^{s-1} \\
& =c \sum_{s=0}^{\infty} \frac{m ! !^{2}}{(m+2 s) ! !^{2}} x^{m+2 s} \mu^{s}-\frac{1}{x} y^{\prime} \\
& =c \frac{m !^{2}}{m ! !^{2}} x^{m}+\mu c \sum_{s=1}^{\infty} \frac{m ! !^{2}}{(m+2 s) ! !^{2}} x^{m+2 s} \mu^{s-1}-\frac{1}{x} y^{\prime} \\
& =c x^{m}+\mu y-\frac{1}{x} y^{\prime}
\end{aligned}
$$

Hence,

$$
y_{p}^{\prime \prime}+\frac{1}{x} y_{p}^{\prime}-\mu y_{p}=c x^{m}
$$

This concludes the proof of Lemma 1.

Finally, using Lemma 1 to obtain $\left\{\Psi_{k, m}\right\}$ in (??) and substituting into (??), we obtain the particular solution for the Fourier-polynomial expansion of $h$ as

$$
\hat{u}_{p}(r, z)=\sum_{k=0}^{K} \sum_{m=0}^{M} \sum_{s=1}^{S} \frac{k ! !^{2}\left(\lambda+m^{2}\right)^{s-1}}{(k+2 s) ! !^{2}} h_{k, m} r^{k+2 s} e^{i m z}
$$

\section{Polynomial Approximation}

As an alternative to the Fourier-polynomial approximation to $h$ in (??), it is possible to use bivariate polynomial approximation to $h$. Such polynomial approximation can be given in the form

$$
\hat{h}(r, z)=\sum_{k=0}^{K} \sum_{m=0}^{M} h_{k, m} r^{k} z^{m}
$$


for sufficiently large $K$ and $M$. In [?], we have shown how to efficiently obtain such approximations.

Denoting by $\Psi_{k, m}$ the particular solution corresponding to $r^{k} z^{m}$ requires solving the PDE

$$
\frac{\partial^{2} \Psi_{k, m}}{\partial r^{2}}+\frac{1}{r} \frac{\partial \Psi_{k, m}}{\partial r}+\frac{\partial^{2} \Psi_{k, m}}{\partial z^{2}}-\mu \Psi_{k, m}=r^{k} z^{m}
$$

By linearity, the particular solution $\hat{u}_{p}$ is given by

$$
\hat{u}_{p}=\sum_{k=0}^{K} \sum_{m=0}^{M} h_{k, m} \Psi_{k, m}
$$

So, it suffices to solve (??) in order to find $\hat{u}_{p}$. To do this, we consider two cases for the power of $r$ in (??).

Case 1: In (??), the right hand side is $r^{2 k} z^{m}$.

To solve (??) with right-hand side $r^{2 k} z^{m}$, we look for a solution in the form

$$
\Psi_{2 k, m}=\sum_{j=0}^{k} \sum_{l=0}^{[m / 2]} A_{j, l} r^{2 k-2 j} z^{m-2 l}
$$

¿From (??), we find the derivatives appearing in (??).

$$
\begin{aligned}
\frac{\partial \Psi_{2 k, m}}{\partial r} & =\sum_{j=0}^{k-1} \sum_{l=0}^{[m / 2]}(2 k-2 j) A_{j, l} r^{2 k-2 j-1} z^{m-2 l} \\
\frac{\partial^{2} \Psi_{2 k, m}}{\partial r^{2}} & =\sum_{j=0}^{k-1} \sum_{l=0}^{[m / 2]}(2 k-2 j)(2 k-2 j-1) A_{j, l} r^{2 k-2 j-2} z^{m-2 l} \\
\frac{\partial^{2} \Psi_{2 k, m}}{\partial r^{2}}+\frac{1}{r} \frac{\partial \Psi_{2 k, m}}{\partial r}=\sum_{j=0}^{k-1} \sum_{l=0}^{[m / 2]}(2 k-2 j)^{2} A_{j, l} r^{2 k-2 j-2} z^{m-2 l} & =\sum_{j=1}^{k} \sum_{l=0}^{[m / 2]}(2 k-2 j+2)^{2} A_{j-1, l} r^{2 k-2 j} z^{m-2 l} \\
\frac{\partial^{2} \Psi_{2 k, m}}{\partial z^{2}} & =\sum_{j=0}^{k} \sum_{l=0}^{[m / 2]-1}(m-2 l)(m-2 l-1) A_{j, l} r^{2 k-2 j} z^{m-2 l-2} \\
& =\sum_{j=0}^{k} \sum_{l=1}^{[m / 2]}(m-2 l+2)(m-2 l+1) A_{j, l-1} r^{2 k-2 j} z^{m-2 l}
\end{aligned}
$$


For convenience, we introduce

$$
A_{-1, l}=0 \quad\left(l=0,1, \ldots,\left[\frac{m}{2}\right]\right) \quad \text { and } \quad A_{j,-1}=0 \quad(j=0,1, \ldots, k) .
$$

¿From (??), equating the coefficients of $r^{2 k-2 j} z^{m-2 l}$, we obtain the system

$$
\begin{gathered}
(2 k-2 j+2)^{2} A_{j-1, l}+(m-2 l+2)(m-2 l+1) A_{j, l-1}-\mu A_{j, l}=\delta_{j, l} \\
\left(j=0,1, \ldots, k \quad \text { and } \quad l=0,1, \ldots,\left[\frac{m}{2}\right]\right)
\end{gathered}
$$

where $\delta_{0,0}=1$ and $\delta_{j, l}=0$ if $(j, l) \neq(0,0)$. For $(j, l)=(0,0)$, we get

$$
A_{0,0}=\frac{-1}{\mu}
$$

Next, by multiplying (??) by $(2 k-2 j) ! !^{2}(m-2 l) ! \mu^{j+l-1},(? ?)$ becomes

$$
\begin{aligned}
& (2 k-2 j+2) ! !^{2}(m-2 l) ! \mu^{j+l-1} A_{j-1, l}+(2 k-2 j) ! !^{2}(m-2 l+2) ! \mu^{j+l-1} A_{j, l-1} \\
& -(2 k-2 j) ! !^{2}(m-2 l) ! \mu^{j+l} A_{j, l} \\
& =\delta_{j, l}(2 k-2 j) ! !^{2}(m-2 l) ! \mu^{j+l-1}
\end{aligned}
$$

Denoting

$$
B_{j, l}=(2 k-2 j) ! !^{2}(m-2 l) ! \mu^{j+l} A_{j, l},
$$

we reduce (??) to

$$
B_{0,0}=(2 k) ! !^{2} m ! A_{0,0}=-\frac{1}{\mu}(2 k) ! !^{2} m !
$$

and (??) (as $(j, l) \neq(0,0))$ to

$$
B_{j-1, l}+B_{j, l-1}=B_{j, l} .
$$

A solution of (??) is given by

$$
B_{j, l}=B_{0,0}\left(\begin{array}{c}
j+l \\
j
\end{array}\right), \quad(j, l) \neq(0,0) .
$$

Indeed,

$$
\begin{aligned}
B_{j-1, l}+B_{j, l-1} & =B_{0,0}\left(\begin{array}{c}
j+l-1 \\
j-1
\end{array}\right)+B_{0,0}\left(\begin{array}{c}
j+l-1 \\
j
\end{array}\right) \\
& =B_{0,0} \frac{(j+l-1) !}{(j-1) ! l !}+B_{0,0} \frac{(j+l-1) !}{j !(l-1) !} \\
& =B_{0,0} \frac{(j+l-1) !}{j ! l !} j+B_{0,0} \frac{(j+l-1) !}{j ! l !} l \\
& =B_{0,0} \frac{(j+l) !}{j ! l !}=B_{j, l}
\end{aligned}
$$


Now, we are ready to get $A_{j, l}$ from (??) using (??) and (??).

$$
A_{j, l}=-\frac{(2 k) !^{2} m !}{(2 k-2 j) ! !^{2}(m-2 l) ! \mu^{j+l+1}}\left(\begin{array}{c}
j+l \\
j
\end{array}\right)
$$

Hence, the solution of (??) is given by

$$
\Psi_{2 k, m}=-\sum_{j=0}^{k} \sum_{l=0}^{[m / 2]} \frac{(2 k) ! !^{2} m ! r^{2 k-2 j} z^{m-2 l}}{(2 k-2 j) ! !^{2}(m-2 l) ! \mu^{j+l+1}}\left(\begin{array}{c}
j+l \\
j
\end{array}\right) .
$$

Case 2: In (??), the right-hand side is $r^{2 k+1} z^{m}$.

In order to solve (??) in this case, we need another important lemma.

Lemma 2 For a given sequence $\left\{a_{n}\right\}$,

$$
\sum_{s=1}^{\infty} \sum_{j=1}^{\infty}\left(\begin{array}{c}
s+l-1 \\
l
\end{array}\right) a_{s+j}=\sum_{s=1}^{\infty}\left(\begin{array}{l}
s+l \\
l+1
\end{array}\right) a_{s+1}
$$

if the series in (??) are convergent.

The proof of Lemma 2 is given in Appendix A.

To solve (??) with right-hand side $r^{2 k+1} z^{m}$, we look for a solution in the form

$$
\Psi_{2 k+1, m}=\sum_{l=0}^{[m / 2]} P_{l}(r) z^{m-2 l}
$$

First, from (??), we calculate the derivatives of $\Psi_{2 k+1, m}$ and then substitute them into the equation

$$
\begin{aligned}
& \frac{\partial^{2}}{\partial r^{2}} \Psi_{2 k+1, m}+\frac{1}{r} \frac{\partial}{\partial r} \Psi_{2 k+1, m}+\frac{\partial^{2}}{\partial z^{2}} \Psi_{2 k+1, m}-\mu \Psi_{2 k+1, m}=r^{2 k+1} z^{m} \\
& \frac{\partial^{2}}{\partial r^{2}} \Psi_{2 k+1, m}+\frac{1}{r} \frac{\partial}{\partial r} \Psi_{2 k+1, m}=\sum_{l=0}^{[m / 2]}\left(\frac{d^{2} P_{l}}{d r^{2}}+\frac{1}{r} \frac{d P_{l}}{d r}\right) z^{m-2 l} \\
& \frac{\partial^{2}}{\partial z^{2}} \Psi_{2 k+1, m}=\sum_{l=0}^{[m / 2]-1}(m-2 l)(m-2 l-1) P_{l}(r) z^{m-2 l-2} \\
&=\sum_{l=1}^{[m / 2]}(m-2 l+2)(m-2 l+1) P_{l-1}(r) z^{m-2 l}
\end{aligned}
$$


Substituting (??) and (??) into (??) and comparing the like terms, we obtain the following system for $P_{l}(r)(l=0,1, \ldots,[m / 2])$.

$$
\frac{d^{2} P_{0}}{d r^{2}}+\frac{1}{r} \frac{d P_{0}}{d r}-\mu P_{0}=r^{2 k+1}
$$

and

$$
\frac{d^{2} P_{l}}{d r^{2}}+\frac{1}{r} \frac{d P_{l}}{d r}-\mu P_{l}=-(m-2 l+2)(m-2 l+1) P_{l-1}, \quad l=1,2, \ldots,[m / 2]
$$

Applying Lemma 1 to (??), we find $P_{0}(r)$ as

$$
P_{o}(r)=\sum_{s=1}^{\infty} \frac{(2 k+1) ! !^{2}}{(2 k+2 s+1) ! !^{2}} r^{2 k+2 s+1} \mu^{s-1}
$$

To get $P_{1}(r)$, we substitute $P_{0}(r)$ from (??) into (??) as $l=1$. (??) becomes

$$
\frac{d^{2} P_{1}}{d r^{2}}+\frac{1}{r} \frac{d P_{1}}{d r}-\mu P_{1}=-m(m-1) \sum_{s=1}^{\infty} \frac{(2 k+1) ! !^{2}}{(2 k+2 s+1) ! !^{2}} r^{2 k+2 s+1} \mu^{s-1}
$$

The solution, $P_{1}$, of (??) is expressed as the following series:

$$
P_{1}(r)=\sum_{s=1}^{\infty} P_{1, s}
$$

where $P_{1, s}$ is a solution of

$$
\frac{d^{2} P_{1, s}}{d r^{2}}+\frac{1}{r} \frac{d P_{1, s}}{d r}-\mu P_{1, s}=-m(m-1) \frac{(2 k+1) ! !^{2}}{(2 k+2 s+1) ! !^{2}} r^{2 k+2 s+1} \mu^{s-1}
$$

Applying Lemma 1 to (??), we find $P_{1, s}$ as

$$
P_{1, s}=-m(m-1) \sum_{j=1}^{\infty} \frac{(2 k+1) !^{2} \mu^{s+j-2}}{(2 k+2 s+2 j+1) !^{2}} r^{2 k+2 s+2 j+1}
$$

Hence, from (??) and (??), we get $P_{1}(r)$ as follows

$$
\begin{aligned}
P_{1}(r) & =-m(m-1) \sum_{s=1}^{\infty} \sum_{j=1}^{\infty} \frac{(2 k+1) !^{2} \mu^{s+j-2}}{(2 k+2 s+2 j+1) ! !^{2}} r^{2 k+2 s+2 j+1} \\
& =-\frac{m !}{(m-2) !} \sum_{s=2}^{\infty} \frac{(2 k+1) !^{2}(s-1) \mu^{s-2}}{(2 k+2 s+1) ! !^{2}} r^{2 k+2 s+1} .
\end{aligned}
$$


Finally, the formula for $P_{1}(r)$ becomes

$$
P_{1}(r)=-\frac{m !}{(m-2) !} \sum_{s=1}^{\infty} \frac{(2 k+1) ! !^{2}\left(\begin{array}{l}
s \\
1
\end{array}\right) \mu^{s-1}}{(2 k+2 s+3) !^{2}} r^{2 k+2 s+3} .
$$

In a similar fashion, using the formula

$$
\sum_{s=1}^{\infty} \sum_{j=1}^{\infty} s a_{s+j}=\sum_{s=2}^{\infty}\left(\begin{array}{l}
s \\
2
\end{array}\right) a_{s}=\sum_{s=1}^{\infty}\left(\begin{array}{c}
s+1 \\
2
\end{array}\right) a_{s+1}
$$

that follows from Lemma 2, omitting the details, we obtain $P_{2}(r)$ as

$$
P_{2}(r)=+\frac{m !}{(m-4) !} \sum_{s=1}^{\infty} \frac{(2 k+1) !^{2}\left(\begin{array}{c}
s+1 \\
2
\end{array}\right) \mu^{s-1}}{(2 k+2 s+5) ! !^{2}} r^{2 k+2 s+5} .
$$

The observation of (??),(??), and (??) leads us to the hypothesis

$$
P_{l}(r)=(-1)^{l} \frac{m !}{(m-2 l) !} \sum_{s=1}^{\infty} \frac{(2 k+1) ! !^{2}\left(\begin{array}{c}
s+l-1 \\
2
\end{array}\right) \mu^{s-1}}{(2 k+2 s+2 l+1) ! !^{2}} r^{2 k+2 s+2 l+1} .
$$

Since (??) has been established for $l=0,1$, and 2 , to prove (??) by the method of the mathematical induction, we need to prove

$$
P_{l+1}(r)=(-1)^{l+1} \frac{m !}{(m-2 l-2) !} \sum_{s=1}^{\infty} \frac{(2 k+1) ! !^{2}\left(\begin{array}{c}
s+l \\
l+1
\end{array}\right) \mu^{s-1}}{(2 k+2 s+2 l+3) ! !^{2}} r^{2 k+2 s+2 l+3} .
$$

assuming that (??) is valid. To do this, we start with (??) with $l$ replaced by $l+1$. Then, (??) becomes

$$
\begin{aligned}
& \frac{d^{2} P_{l+1}}{d r^{2}}+\frac{1}{r} \frac{d P_{l+1}}{d r}-\mu P_{l+1} \\
& =-(m-2 l)(m-2 l-1) P_{l} \\
& =\frac{(-1)^{l+1} m !}{(m-2 l-2) !} \sum_{s=1}^{\infty} \frac{(2 k+1) ! !^{2}\left(\begin{array}{c}
s+l-1 \\
l
\end{array}\right) \mu^{s-1}}{(2 k+2 s+2 l+1) ! !^{2}} r^{2 k+2 s+2 l+1} .
\end{aligned}
$$

As before, we introduce the functions $P_{l+1, s}$ that are solutions of

$$
\frac{d^{2} P_{l+1, s}}{d r^{2}}+\frac{1}{r} \frac{d P_{l+1, s}}{d r}-\mu P_{l+1, s}=\frac{(-1)^{l+1} m !}{(m-2 l-2) !} \frac{(2 k+1) ! !^{2}\left(\begin{array}{c}
s+l-1 \\
l
\end{array}\right) \mu^{s-1}}{(2 k+2 s+2 l+1) ! !^{2}} r^{2 k+2 s+2 l+1} .
$$

After (??) are solved, $P_{l+1}(r)$ is found by

$$
P_{l+1}(r)=\sum_{s=1}^{\infty} P_{l+1, s}
$$


Applying Lemma 1 to (??), we find

$$
P_{l+1, s}=\frac{(-1)^{l+1} m !}{(m-2 l-2) !} \sum_{j=1}^{\infty} \frac{(2 k+1) !^{2}\left(\begin{array}{c}
s+l-1 \\
l
\end{array}\right) \mu^{s+j-2}}{(2 k+2 s+2 l+2 j+1) ! !^{2}} r^{2 k+2 s+2 l+2 j+1}
$$

Next we substitute (??) into (??) and apply Lemma 2 to get $P_{l+1}(r)$.

$$
\begin{aligned}
P_{l+1}(r) & =\frac{(-1)^{l+1} m !}{(m-2 l-2) !} \sum_{s=1}^{\infty} \sum_{j=1}^{\infty} \frac{(2 k+1) !^{2}\left(\begin{array}{c}
s+l-1 \\
l
\end{array}\right) \mu^{s+j-2}}{(2 k+2 s+2 l+2 j+1) ! !^{2}} r^{2 k+2 s+2 l+2 j+1} \\
& =\frac{(-1)^{l+1} m !}{(m-2 l-2) !} \sum_{s=1}^{\infty} \frac{(2 k+1) ! !^{2}\left(\begin{array}{c}
s+l \\
l+1
\end{array}\right) \mu^{s-1}}{(2 k+2 s+2 l+3) ! !^{2}} r^{2 k+2 s+2 l+3}
\end{aligned}
$$

This is equivalent to $P_{l+1}(r)$ satisfying (??). This also concludes the proof of our hypothesis, (??), by the method of mathematical induction.

Next, we obtain $u_{2 k+1, m}$, the particular solution of (??) by substituting (??) into (??).

$$
\Psi_{2 k+1, m}=\sum_{l=0}^{[m / 2]} \sum_{s=1}^{\infty} \frac{(-1)^{l} m !}{(m-2 l) !} \frac{(2 k+1) !^{2}\left(\begin{array}{c}
s+l-1 \\
l
\end{array}\right) \mu^{s-1}}{(2 k+2 s+2 l+1) ! !^{2}} r^{2 k+2 s+2 l+1} z^{m-2 l}
$$

Finally, the solution of (??) in the case of (??) is given by revisiting of (??).

$$
u_{p}(r, z)=\sum_{k=0}^{\infty} \sum_{m=0}^{\infty} h_{2 k, m} \Psi_{2 k, m}+\sum_{k=0}^{\infty} \sum_{m=0}^{\infty} h_{2 k+1, m} \Psi_{2 k+1, m}
$$

(??) yields

$$
u_{p}(r, z) \approx \hat{u}_{p}(r, z)=\sum_{k=0}^{[K / 2]} \sum_{m=0}^{M} h_{2 k, m} \Psi_{2 k, m}+\sum_{k=0}^{[K / 2]} \sum_{m=0}^{M} h_{2 k+1, m} \Psi_{2 k+1, m}
$$

In (??) and (??), $\Psi_{2 k, m}$ is given by (??), and $\Psi_{2 k+1, m}$ is given by (??).

\section{$6 \quad$ Numerical Results}

Since the particular solution is not unique, it is difficult to validate the numerical accuracy of the approximate particular solution $\hat{u}_{p}$ in (??). Notice that the numerical accuracy of $\hat{u}_{p}$ depends on the evaluation of $\Psi_{2 k, m}$ and $\Psi_{2 k+1, m}$ in (??). In this section, we numerically evaluate $\Psi_{2 k, m}$ and $\Psi_{2 k+1, m}$ using the analytic formulae given by (??) and (??). The numerical evaluation of $\Psi_{2 k, m}$ in (??) is straightforward. It is not trivial for the case of $\Psi_{2 k+1, m}$ which involves an infinite series, as shown in (??). Furthermore, the term $\mu^{s-1}$ in (??) is in the numerator and could grow significantly large when the wave number and $s$ 
become large. This may present difficulties in the numerical evaluation due to round-off error.

The numerical evaluation of $\Psi_{2 k, m}$ and $\Psi_{2 k+1, m}$ can be directly performed by using MATHEMATICA. For instance, $\left(\begin{array}{c}s+l-1 \\ l\end{array}\right)$ can be evaluated using Binomial[s+l-1,1], and $[\mathrm{m} / 2]$ can be replaced by IntegerPart $[\mathrm{m} / 2]$. The other parts, such as summation and factorial, can be used as they are. To validate the numerical evaluation of $\Psi_{2 k, m}$ and $\Psi_{2 k+1, m}$, we compute the residue

$$
\left(\frac{\partial^{2}}{\partial r^{2}}+\frac{1}{r} \frac{\partial}{\partial r}+\frac{\partial^{2}}{\partial z^{2}}-\mu\right) \Psi-r^{k} z^{m} .
$$

Let

$$
L=\frac{\partial^{2}}{\partial r^{2}}+\frac{1}{r} \frac{\partial}{\partial r}+\frac{\partial^{2}}{\partial z^{2}}-\mu .
$$

We denote $e_{\mu}(r, z)$ and $\hat{e}_{\mu}(r, z)$ as the absolute maximum residues as follows.

$$
\begin{aligned}
& e_{\mu}(r, z)=\max _{1 \leq k, m \leq 5}\left|L \Psi_{2 k+1, m}-r^{2 k+1} z^{m}\right| \\
& \hat{e}_{\mu}(r, z)=\max _{1 \leq k, m \leq 5}\left|L \Psi_{2 k, m}-r^{2 k} z^{m}\right|
\end{aligned}
$$

To validate the accuracy of the particular solution for various $(r, z)$, we fix $k$ and $m$ and compute the absolute maximum residue $E_{\mu}(k, m)$ and $\hat{E}_{\mu}(k, m)$ at 100 evenly distributed points in $[-1,1] \times[-1,1]$; i.e.,

$$
\begin{aligned}
& E_{\mu}(k, m)=\max _{-1 \leq r, z \leq 1}\left|L \Psi_{2 k+1, m}-r^{2 k+1} z^{m}\right| \\
& \hat{E}_{\mu}(k, m)=\max _{-1 \leq r, z \leq 1}\left|L \Psi_{2 k, m}-r^{2 k} z^{m}\right|
\end{aligned}
$$

We first examine the numerical results of the residue errors for $\Psi_{2 k, m}$. In Table ??, we compute the absolute maximum residue $\hat{e}_{1000}(r, z)$ at three selected points. This means, for the fixed values $\mu=1000$ and $(r, z)$, we compute the absolute residue of 25 different ordered pairs of $k$ and $m$. Note that large values of $\mu$ will not affect the numerical accuracy of $\Psi_{2 k, m}$, since $\mu$ appears in the denominator of (??).

Table 1: The absolute maximum residue $\hat{e}_{1000}(r, z)$ at three selected points.

\begin{tabular}{|l|l|}
\hline$(r, z)$ & $\hat{e}_{1000}(r, z)$ \\
\hline$(0.25,0.25)$ & $2.168 E-19$ \\
\hline$(0.5,0.5)$ & $1.734 E-18$ \\
\hline$(0.75,0.75)$ & $5.551 E-17$ \\
\hline
\end{tabular}


Table 2: The absolute maximum residues $E_{1000}(k, m)$ and $\hat{E}_{1000}(k, m)$.

\begin{tabular}{|l|l|l|l|}
\hline$k$ & $m$ & $E_{1000}(k, m)$ & $\hat{E}_{1000}(k, m)$ \\
\hline 1 & 1 & $3.30 E-9$ & $1.11 E-16$ \\
\hline 1 & 2 & $4.63 E-8$ & $1.11 E-16$ \\
\hline 2 & 2 & $6.43 E-10$ & $2.77 E-17$ \\
\hline 2 & 3 & $1.44 E-9$ & $5.55 E-17$ \\
\hline
\end{tabular}

Next, we fix the values of $\mu, k$, and $m$, then arrange $(r, z)$ through 100 test points in $[-1,1] \times[-1,1]$. The absolute maximum residues $E_{1000}(k, m)$ and $\hat{E}_{1000}(k, m)$ are shown in Table ??. It is clear that $\Psi_{2 k+1, m}$ is much more difficult to evaluate than $\Psi_{2 k, m}$.

In Figure ??, we show the numerical accuracy of $\Psi_{2 k+1, m}$ for various values of $s$ in (??) using $\mu=1000$ at three selected points. For larger values of $r$ and $z$, more terms from the infinite series are required and the overall accuracy is smaller compared to small values of $r$ and $z$. The contribution of the errors in the evaluation of $\Psi_{2 k+1, m}$ largely result from round-off error instead of truncation error. In Figure ??, we test the numerical accuracy of $\Psi_{2 k+1, m}$ at $(r, z)=(0.75,0.75)$ for $\mu=200,500,1000$ and various $s$ terms in (??). We observe that higher values of $\mu$ result in higher residue error. This means the truncation error is more pronounced for higher wave numbers $\mu$, and thus, a larger number of $s$ terms is required.

Figure 2: The absolute maximum residue $e_{1000}(r, z)$ versus the number of terms used at three select points for the odd case.

Figure 3: The absolute maximum residue $e_{\mu}(0.75,0.75)$ versus the number of terms used for the odd case and various $\mu$.

Form Figures ?? and ??, we notice that the absolute maximum residue remains the same when $s>40$. This implies that the round-off error dominates the truncation error. When $s$ is sufficiently large, no further numerical improvement can be achieved unless the round-off error can be properly taken care of. As we know, MATHEMATICA is capable of carrying the numerical computation with infinite precision. We notice that the analytic formulation of $\Psi_{2 k, m}$ in (??) and $\Psi_{2 k+1, m}$ in (??) is ideal for the task of errorfree evaluation which can be achieved by taking $r$ and $z$ as rational numbers instead of decimal numbers. As a result of error-free computation, the effect of round-off error becomes nil. In particular, $\Psi_{2 k, m}$ can be obtained error-free; i.e., $\hat{e}_{\mu}(r, z)=\hat{E}_{\mu}(k, m)=0$ 
for all of $\mu, r, z, k$, and $m$. As shown in Table ??, similar results for $\Psi_{2 k+1, m}$ can also be obtained. Since no round-off error is encountered, high precision evaluation of $\Psi_{2 k+1, m}$ can be achieved by increasing the number of terms in the infinite series in (??).

Table 3: Error-free evaluation of absolute residues for some selected values.

\begin{tabular}{|c|c|c|c|c|c|c|}
\hline$r$ & $z$ & $k$ & $m$ & $\mu$ & $s$ & Absolute Residues \\
\hline \multirow[t]{4}{*}{$1 / 4$} & \multirow[t]{4}{*}{1} & \multirow[t]{4}{*}{1} & \multirow[t]{4}{*}{1} & \multirow[t]{4}{*}{2000} & 15 & $9.96 E-8$ \\
\hline & & & & & 35 & $1.13 E-34$ \\
\hline & & & & & 55 & $1.58 e-71$ \\
\hline & & & & & 75 & $1.32 E-114$ \\
\hline \multirow[t]{4}{*}{$5 / 4$} & \multirow[t]{4}{*}{1} & \multirow[t]{4}{*}{2} & \multirow[t]{4}{*}{3} & \multirow[t]{4}{*}{2000} & 65 & $3.88 E-2$ \\
\hline & & & & & 75 & $1.82 E-10$ \\
\hline & & & & & 95 & $2.37 E-30$ \\
\hline & & & & & 115 & $8.34 E-54$ \\
\hline \multirow[t]{4}{*}{$3 / 5$} & \multirow[t]{4}{*}{$4 / 3$} & \multirow[t]{4}{*}{4} & \multirow[t]{4}{*}{5} & \multirow[t]{4}{*}{5000} & 65 & $1.62 E-24$ \\
\hline & & & & & 75 & $1.76 E-35$ \\
\hline & & & & & 85 & $1.57 E-47$ \\
\hline & & & & & 95 & $1.51 E-60$ \\
\hline
\end{tabular}

\section{Conclusions}

In this paper, we focus our work on the derivation of the axisymmetric particular solution of Helmholtz-type equations. Fourier polynomial approximation and polynomial approximation are discussed. Coupled with boundary methods [?, ?] and various integral transformations and time-stepping methods, transient solutions of the time-dependent problems can be obtained. Using the method of fundamental solutions or Trefftz method as the boundary method, we can achieve meshless computation.

The particular solution derived in this paper is most suitable for symbolic computation. For floating number operations, the users need to aware the effect of round-off errors. In such case, the higher machine precision is desirable.

We will continue to pursuit our research by extending our derived particular solution to solve various time-dependent problems. We expect our approach will provide an efficient algorithm for solving various types of axisymmetric time-dependent problems.

Acknowledgement: The first and third authors acknowledge the support of NATO Collaborative Linkage Grant under Reference PST.CLG.980398. 


\section{References}

[1] P. W. Partridge, C. A. Brebbia and C.A., L.C. Wrobel, The Dual Reciprocity Boundary Element Mehod, Computational Mechanics Publications, Southampton and Elsevier, London, 1992.

[2] E. Kita and N. Kamiya, Trefftz method: An overview. Advances in Engineering software, 24, 1-12.

[3] J.M. Melenk, I. Babuška, Approximation with harmonic and generalized harmonic polynomials in the partition of unity method, Computer Assisted Mechanical Engineering Sciences, 4, 607-633, 1995.

[4] S. Langdon, Domain Embedding Boundary Integral Equation Methods and Parabolic PDEs, Ph.D. Thesis, Universtiy of Bath, U.K., 1999.

[5] J. Su, B. Tabarrrok, A time-marching integral equations method for unsteady state problems, Computer Methods in Applied Mechanics and Engineering, 142, 203-214, 1997.

[6] Kaichun Wang, BEM simulation for glass parisons, Ph.D. thesis, Eindhoven Technical University, the Netherlands, 2002.

[7] Hokwon A. Cho, M.A. Golberg, A.S. Muleshkov, and Xin Li, Trefftz Methods for Time Dependent Partial Differential Equations, CMC, 1, 1-37, 2004.

[8] M.A. Golberg and C.S. Chen, An efficient meshfree method for nonlinear reactiondiffusion equations, CMES, 2(1), 87-95, 2001.

[9] D. Nardini and C.A. Brebbia, A new approach to free vibration analysis using boundary elements, In: Boundary Element Methods in Engineering, Proc. 4th Int. Sem., Southamton, C.A. Brebbia, editor, Springer-Verlag, 312-326, 1982.

[10] Božidar Šarler, Axisymmetric augmented thin plate splines, Engineering Analysis with Boundary Elements, 21(1), 81-85, 1998.

[11] C.S. Chen, A.S. Muleshkov, M.A. Golberg, and R.M.M. Mattheij, A mesh free approach to solving the axisymmetric Poisson's equation, Numerical Methods for Partial Differential Equations, to appear in 2005.

[12] M. A. Golberg, A. S. Muleshkov, C. S. Chen, and A. D.-H. Cheng, Polynomial particular solutions for some partial differential operators, Numerical Methods for Partial Differential Equations, 19, 112-133, 2003.

[13] K.H. Park, A BEM formulation for inhomogeneous integrals, Applied Mathematical Modeling, 27, 293-306, 2003. 
[14] F. Bai, W.Q. Lu, The selection and assemblage of approximation functions and disposal of its singularity in axisymmetric DRBEM for heat transfer problems, Engineering Analysis with Boundary Elements, 28, 955-965, 2004.

[15] H. Stehfest, Algorithm 368: Numerical Inversion of the Laplace Transform, Communincations of the ACM, 13, 47-49, 1970.

[16] G. Fairweather, A. Karageorghis and P. A. Martin, The method of fundamental solutions for scattering and radiation problems, Engineering Analysis with Boundary Elements, 27, 759-769, 2003.

[17] A. Karageorghis and G. Fairweather, The method of fundamental solutions for axisymmetric acoustic scattering and radiation problems, Journal of the Acoustical Society of America, 104, 3212-3218, 1998.

\section{Appendix A}

Proof of Lemma 2

For a given sequence $\left\{a_{n}\right\}$,

$$
\sum_{s=1}^{\infty} \sum_{j=1}^{\infty}\left(\begin{array}{c}
s+l-1 \\
l
\end{array}\right) a_{s+j}=\sum_{s=1}^{\infty}\left(\begin{array}{l}
s+l \\
l+1
\end{array}\right) a_{s+1}
$$

if the series in (??) are convergent.

Proof.

$$
\begin{aligned}
& \sum_{s=1}^{\infty} \sum_{j=1}^{\infty}\left(\begin{array}{c}
s+l-1 \\
l
\end{array}\right) a_{s+j} \\
& =\left(\begin{array}{l}
l \\
l
\end{array}\right) a_{2}+\left(\begin{array}{c}
l+1 \\
l
\end{array}\right) a_{3}+\left(\begin{array}{c}
l+2 \\
l
\end{array}\right) a_{4}+\ldots+\left(\begin{array}{l}
l \\
l
\end{array}\right) a_{3}+\left(\begin{array}{c}
l+1 \\
l
\end{array}\right) a_{4}+\left(\begin{array}{c}
l+2 \\
l
\end{array}\right) a_{5}+\ldots \\
& +\left(\begin{array}{l}
l \\
l
\end{array}\right) a_{4}+\left(\begin{array}{c}
l+1 \\
l
\end{array}\right) a_{5}+\left(\begin{array}{c}
l+2 \\
l
\end{array}\right) a_{6}+\ldots \\
& =\left(\begin{array}{l}
l \\
l
\end{array}\right) a_{2}+\left[\left(\begin{array}{l}
l \\
l
\end{array}\right)+\left(\begin{array}{c}
l+1 \\
l
\end{array}\right)\right] a_{3}+\left[\left(\begin{array}{l}
l \\
l
\end{array}\right)+\left(\begin{array}{c}
l+1 \\
l
\end{array}\right)+\left(\begin{array}{c}
l+2 \\
l
\end{array}\right)\right] a_{4}+\ldots \\
& +\left[\left(\begin{array}{l}
l \\
l
\end{array}\right)+\left(\begin{array}{c}
l+1 \\
l
\end{array}\right)+\ldots+\left(\begin{array}{c}
l+s-1 \\
l
\end{array}\right)\right] a_{s+1}+\ldots \\
& =\sum_{s=1}^{\infty} a_{s+1} \sum_{j=1}^{\infty}\left(\begin{array}{c}
l+j-1 \\
l
\end{array}\right)
\end{aligned}
$$


Denoting

$$
T_{s}=\sum_{j=1}^{s}\left(\begin{array}{c}
l+j-1 \\
l
\end{array}\right)
$$

we prove

$$
T_{s}=\left(\begin{array}{l}
s+l \\
l+1
\end{array}\right)
$$

by using the method of mathematical induction. Since

$$
T_{1}=\sum_{j=1}^{1}\left(\begin{array}{c}
l+j-1 \\
l
\end{array}\right)=\left(\begin{array}{l}
l \\
l
\end{array}\right)=1=\left(\begin{array}{l}
1+l \\
l+1
\end{array}\right)
$$

it is enough to prove

$$
T_{n+1}=\left(\begin{array}{c}
n+1+l \\
l+1
\end{array}\right)
$$

if we assume

$$
T_{n}=\left(\begin{array}{c}
n+l \\
l+1
\end{array}\right)
$$

Indeed,

$$
\begin{aligned}
T_{n+1} & =\sum_{j=1}^{n+1}\left(\begin{array}{c}
l+j-1 \\
l
\end{array}\right)=\sum_{j=1}^{n}\left(\begin{array}{c}
l+j-1 \\
l
\end{array}\right)+\left(\begin{array}{c}
n+l \\
l
\end{array}\right) \\
& =T_{n}+\left(\begin{array}{c}
n+l \\
l
\end{array}\right)=\left(\begin{array}{c}
n+l \\
l+1
\end{array}\right)+\left(\begin{array}{c}
n+l \\
l
\end{array}\right) \\
& =\frac{(n+l) !}{(l+1) !(n-1) !}+\frac{(n+l) !}{l ! n !} \\
& =n \frac{(n+l) !}{(l+1) ! n !}+(l+1) \frac{(n+l) !}{(l+1) ! n !} \\
& =\frac{(n+l) !(n+l+1)}{(l+1) ! n !}=\left(\begin{array}{c}
n+1+l \\
l+1
\end{array}\right)
\end{aligned}
$$

Going back to the beginning of the proof, we get

$$
\sum_{s=1}^{\infty} \sum_{j=1}^{\infty}\left(\begin{array}{c}
s+l-1 \\
l
\end{array}\right) a_{s+j}=\sum_{s=1}^{\infty} a_{s+1} T_{s}=\sum_{s=1}^{\infty}\left(\begin{array}{c}
s+l \\
l+1
\end{array}\right) a_{s+1} .
$$

This concludes the proof of Lemma 2. 